Grasso, Marzio, Azzouz, Lyes, Ruiz-Hincapie, Paula, Zarrelli, Mauro and Ren, Guogang (2018) Effect of temperature on the mechanical properties of 3D-printed PLA tensile specimens. Rapid Prototyping Journal, 24(8), pp. 1337-1346. ISSN (print) 1355-2546.

https://doi.org/10.1108/RPJ-04-2017-0055 


\section{Effect of Temperature on the Mechanical Properties of 3D-Printed PLA Tensile Specimens}

Marzio Grasso, Lyes Azzouz and Paula Ruiz-Hincapie

School of Engineering and Technology, University of Hertfordshire, Hatfield, UK

Mauro Zarrelli

IPCB - Institute of Polymers, Composites and Biomaterials, CNR - Research National Council, Portici, Italy, and Guogang Ren

School of Engineering and Technology, University of Hertfordshire, Hatfield, UK

\section{Introduction}

Additive manufacturing (AM), also known as "tool-less" manufacturing, can be quickly executed and has progressed substantially in recent years. This technology significantly reduces material waste by eliminating unnecessary processes and assembly steps ( $\mathrm{Li}, \mathrm{Wu}$ et al. 2016). Currently used techniques include fused deposition modelling (FDM), stereo-lithography, selective laser sintering, syringe extrusion and fused filament deposition (FFD) (Gibson, Rosen et al. 2010).FDM is one of the most used 3D printing techniques of thermoplastic polymers. This process is based on the deposition of semi-molten filaments to create an object as a composition of several overlapping layers; of which each layer is composed of filaments laid out according to a predetermined rastering configuration. A significant advantage of utilising FDM technology is the potential to create complex and specific shapes.. The process is currently adopted in bio-engineering, mainly for the manufacturing of tissue engineering scaffolds (Visscher, Bos et al. 2016, Hong, Newman et al. 2017, Shakor, Sanjayan et al. 2017). Additionally, the automotive sector uses this technology to produce small components (Keles, Blevins et al. 2017). However, it is gaining more attention in several other fields including energy harvesting (Bito, Bahr et al. 2017).

Conventionally, fossil fuel based plastics have been widely adopted by several industries. However, over the last several decades eco-friendly, biodegradable and medical grade polymer materials have received considerable attention due to improvements in their manufacturing processes and the possibility of creating blends to achieve more advantageous materials. Among the vast myriad of 
biodegradable materials, Polylactic Acid (PLA) has emerged as one of the preferred candidates for 3D printing given its many favourable properties including biodegradability, biocompatibility, composability and recyclability (Farah, Anderson et al. 2016). Additionally, PLA is widely used in emerging technologies such as 3D printing along with others polymers namely Acrylonitrile Butadiene Styrene (ABS), PolyCarbonate (PC), and PolyVinyl Alcohol (PVA) (Torres, Cotelo et 
al. 2015). PLA is characterized by low operating temperatures, specifically the glass transition temperature $\mathrm{T}_{\mathrm{g}}$ and the melting temperature $\mathrm{Tm}$ (Lanzotti, Grasso et al. 2015) which are preferable characteristic for both the softening during the "deposition" stage $\left(\mathrm{T}_{\mathrm{g}}\right)$ and avoiding localised degradation process due to hot spot in complex geometry $\left(\mathrm{T}_{\mathrm{m}}\right)$. The fundamental issue in designing 3D printed components is the identification of strength and stiffness (Qin, Compton et al. 2015). Several authors have carried out experimental tests to investigate the mechanical behaviour. For instance, in (de Obaldia, Jeong et al. 2015) it is suggested that 3D printed materials exhibit highly anisotropic behaviour in both their stiffness and strength properties. Other studies have also reported that an increase in the layer thickness reduces those same features (Durgun and Ertan 2014, Impens and Urbanic 2016, Torres, Cole et al. 2016). In addition, fracture surface investigations have been used to explain the high variability in the aforementioned properties; this technique relies on the identification of physical landmarks that correlate failure mechanisms with loading regimes (Bellini and Güçeri 2003, Moroni, De Wijn et al. 2006, Lanzotti, Grasso et al. 2015). The effects of the infill orientation have been previously discussed by other authors; these orientation arrangements include unidirectional bead lay-up and criss-cross bead lay-up in which the beads of adjacent layers are separated by 90॰ (Thomas and Renaud 2003). In both of these cases, the effect of the infill orientation on the stiffness and strength of the component have been found similar to that of composite materials (Rodríguez, Thomas et al. 2001). Nevertheless, the main difference between $3 \mathrm{D}$ printed parts and composite materials is the shear stress transfer mechanism among adjacent layers. In the case of 3D printed components, adjacent beads, from a layer-upon-layer configuration viewpoint, are blended rather than attached via a substrate material as typically encountered in fibre-reinforced polymers. Therefore, the overlapping surface in 3D printed components could be varied by modifying the parameters related to the deposition phase such as temperature, deposition rate or contact pressure. The extension of bonding surfaces play an essential role for the 3D printed 
parts, by withstanding the tangential load acting on the element while in long fibre-reinforced polymer composite, the shear performance is related to the strength of the matrix mainly.

Attempts to adopt the interlaminar shear strength as a failure criterion for 3D printed parts have been made by considering empirical coefficients to account for the effect of the overlapping surfaces (Thomas and Renaud 2003). However, inconsistencies continue to appear when comparing numerical results to experimental data of 3D printed parts (Bikas, Stavropoulos et al. 2016). In many cases, strength and stiffness of 3D printed parts have been investigated under simplistic conditions such as static loads at ambient temperature while neglecting the effect of time and temperature associated with the deposition period of the final part. Noteworthy, PLA undergoes physical changes during printing and whilst in service due to varying temperatures, namely glass transition, between $50^{\circ} \mathrm{C}$ and $70^{\circ} \mathrm{C}$ (Senatov, Niaza et al. 2016) and other thermal transitions.

Therefore this is a critical factor that must be considered in the production and use of PLA in a variety of applications within aerospace, automotive and medical sectors. AM implementation has seen a significant market growth over the last decade in several industries and research sectors. The market value of 3D printed components in the automotive sector ranked as the highest globally, has been estimated at 7000 million US dollars by 2025 while the medical applications sector is listed as the third fastest growing industry utilising such technologies (Feng, Wu et al. 2014). Despite the rapid growth in the number of AM-related publications over the last five years, approximately 3500 per year, there is still a gap in the open literature regarding the effect of temperature on the mechanical properties of 3D printed components. Furthermore, in-situ mechanical testing over a range of in-service temperatures is a significant factor in identifying failure modes, that is, whether the fracture is brittle or ductile. Considering the viscoelastic nature of polymeric materials, the elastic proportion of stress-strain curve followed by a sudden drop in stress with no further extension is said to be a brittle fracture failure. However, in ductile dominant failure, the elastic and plastic proportion are merged with a decreased value of maximum stress and a prolonged extension 
which is sometimes infinite. These effects are controlled by the addition of plasticizers or temperature variation (Kendall and Siviour 2014). Therefore, it stands as mandatory to investigate the impact of temperature on the strength and stiffness of the 3D printed materials; ultimately benefiting the selection of $3 \mathrm{D}$ printing processing parameters or conditions, rather than just being a geometric specification, according to the required applications.

In this work, the thermal, mechanical properties of 3D printed PLA specimens has been investigated by considering different raster angles and environmental temperatures $20^{\circ} \mathrm{C}-60^{\circ} \mathrm{C}$. The heatinduced variations of the feeding material have also been studied by performing mechanical tests on the plain, referring to the filaments before the extrusion process takes place, and the extruded filaments in tensile mode configuration. This was done with the aim of assessing potential property changes related to the physical variation of the polymeric material. Thus, the ultimate tensile strength, Young's modulus, strain at failure and stress at failure of these 3D-printed samples were determined at different temperatures to evaluate final material performance along three bead orientations, namely $0 \mathrm{o} / 90 \mathrm{o},-30 / 60 \mathrm{o}, \pm 45 \mathrm{o}$, while the temperature was kept constant over several values ranging between $20^{\circ} \mathrm{C}$ and $60^{\circ} \mathrm{C}$.

\section{Materials and Methods}

\subsection{PLA Filament Sample Preparation and Mechanical Characterisation}

In order to assess the potential physical change in the feeding material due to the time-temperature profile undergone by the system during the deposition stage, preliminary mechanical tests were carried out on plain and on freely extruded PLA filament samples, that is those that are left to run for a few seconds as part of the filament loading process and cleared from the build plate before printing takes place. Each test was repeated five times for statistical significance. In Table 1, the plain and freely extruded wire-like samples are reported along with the considered temperatures and the number of performed test repetitions. 
Table 1 - Experimental plan for the PLA filament

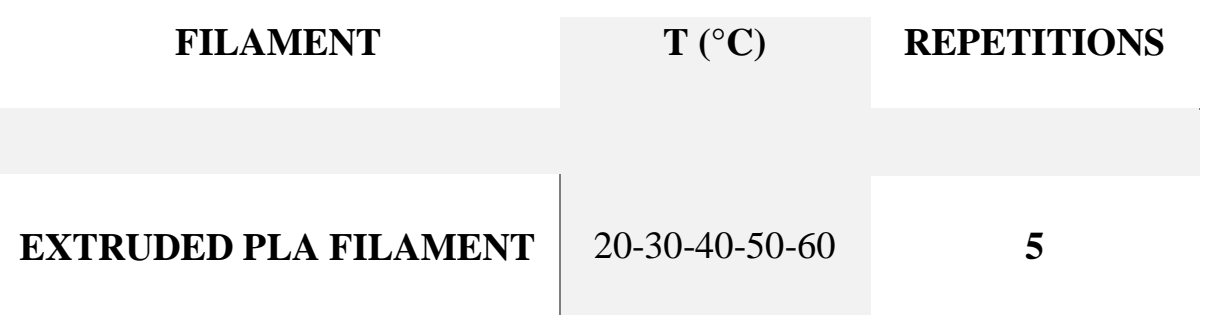

PLA filaments, both plain and extruded, were tested by using a Dynamic Mechanical Analyser, model Q800 DMA (TA Instruments Italy), equipped with a fibre tension clamp. The tension tests were force controlled up to the maximum $18 \mathrm{~N}$ by using a force ramp of $1 \mathrm{~N} / \mathrm{min}$. Nominal dimensions of the cylindrical wire-like filament were $15 \mathrm{~mm}$ in length and $1.75 \mathrm{~mm}$ and $0.60 \mathrm{~mm}$ in diameter for plain and extruded filaments respectively.

\subsection{PLA Standard Specimen Preparation and Testing}

Tensile mechanical tests were carried out on standard PLA 3D-printed samples (see Figure 1) to characterise specimens with three different bead orientations, namely $0 \circ / 90 \circ,-30 \circ /$ $60 \circ$ and $\pm 45 \circ$, over a range of different temperatures from room temperature $\left(20^{\circ} \mathrm{C}\right)$ to $60^{\circ} \mathrm{C}$ with a step increment of $10^{\circ} \mathrm{C}$. To avoid the failure of the specimens outside the gauge length, likely brought about by the approximation of curvatures as a result of the discretised geometry produced at the termination FDM roads, the geometry proposed in (Lanzotti, Grasso et al. 2015) was adopted. In particular, this geometry has a fillet with a parabolic curve drawn as an arc (with a radius of $1,000 \mathrm{~mm}$ ) tangent to the start of the linear segment at the reduced section of the specimen. Specimen dimensions are specified in Figure 1. 


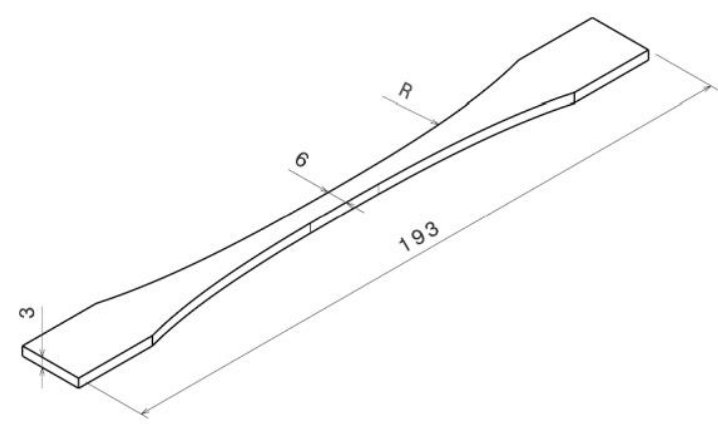

Figure 1 - Standard sample nominal dimensions. All dimension in millimetres.

As reported by (Ahn, Montero et al. 2002) this geometry reduces premature shear failure at the stress concentrations caused by the discretization of large radii along the length of the specimen; this is particularly evidenced at the termination of FDM roads or insets to approximate large radii. The geometry of the 3D samples and the stereolithographic (STL) files were generated with the CAD software CATIA V5 (Dassualt Systemes, Velizy-Villacoublay, France). As illustrated in Figure 2 , the specimens were printed in the $\mathrm{x}-\mathrm{y}$ plane and rotated about the $\mathrm{z}$-axis with a raster angle of $0^{\circ}$, $30^{\circ}$ and $45^{\circ}$ to generate the different infill orientations $0^{\circ} / 90^{\circ},-30^{\circ} / 60^{\circ}$ and $\pm 45^{\circ}$ respectively; see Figure 3.

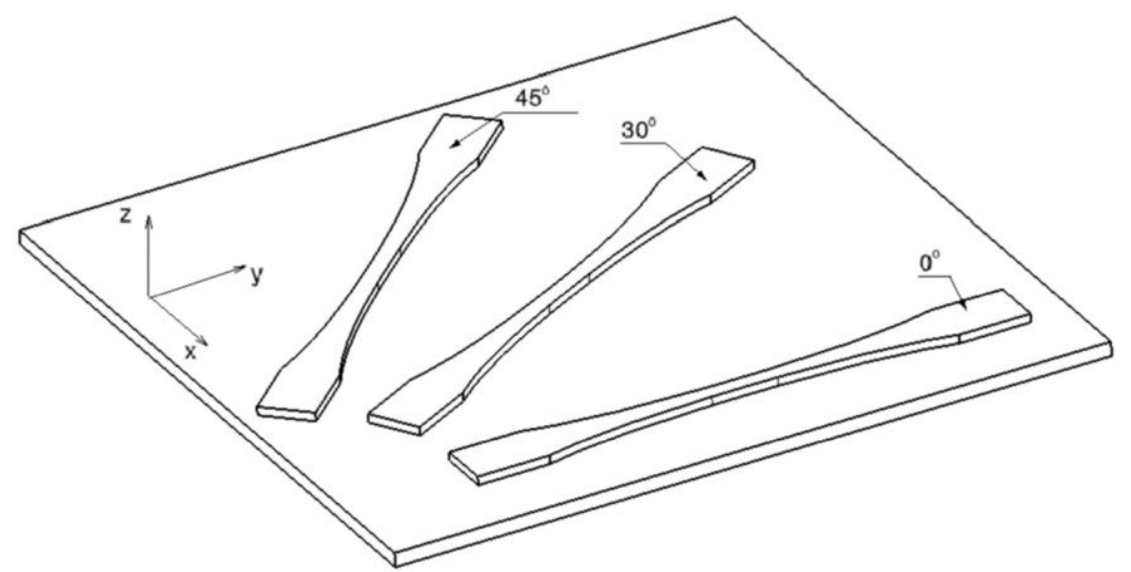

Figure 2 - Schematic arrangement of the different raster angle values $\left(0^{\circ}-30^{\circ}-45^{\circ}\right)$ adopted to print the three different infill orientations 
A total of 75 standard tensile specimens were produced and tested, within which, each of the three different infill orientations was tested at five different temperatures from 20 to $60{ }^{\circ} \mathrm{C}$. The tensile tests were repeated five times to ensure statistical significance. Table 2 reports the experimental plan for all PLA samples manufactured at different infill orientations and temperatures. The ID indicated in Table 2 is composed of two parts; the first signifies the temperature value (T1, T2 and $\mathrm{T} 3)$ and the second signifies the considered orientation (O1, O2 and $\mathrm{O} 3)$.

Table 2 Experimental plan

\begin{tabular}{c|ccc} 
ID & INFILL & T & REPETITIONS \\
& ORIENTATION & $\left({ }^{\circ} \mathbf{C}\right)$ & \\
T201 & $0^{\circ} / 90^{\circ}$ & 30 & 5
\end{tabular}


The MakerBot Replicator Desktop 3D printer (MakerBot Industries, Brooklyn, USA) with a 0.4-mm diameter nozzle was used to produce the tensile test specimens. The values of the primary geometry control parameters including layer thickness, number of shells or perimeters and overlapping are shown in Table 3. The aforementioned parameters, together with the printing process parameters such as feed rate, travel speed and print temperature, were tuned. This tuning was based on the quality of the print out specimens in terms of dimensions, smoothness of the outer surfaces and the volume of material needed to print the samples.

Table 3 Printing parameters implemented in the MakerBot Replicator

\begin{tabular}{c|c|c}
\hline Parameters & Value & Unit \\
\hline Layer height & $\mathbf{0 . 1 8}$ & $\mathbf{m m}$ \\
\hline Infill Layer Height & $\mathbf{0 . 1 8}$ & $\mathbf{m m}$ \\
\hline Number of shells & 2 & - \\
\hline Feed rate & 1 & $\mathrm{~mm} / \mathbf{s}$ \\
\hline Travel feed rate & 10 & $\mathrm{~mm} / \mathbf{s}$ \\
\hline Print temperature & 215 & $\circ \mathrm{C}$ \\
\hline Filament diameter & 1.75 & $\mathbf{m m}$ \\
\hline
\end{tabular}




\begin{tabular}{c|c|c} 
Nozzle diameter & $\mathbf{0 . 4}$ & $\mathbf{m m}$ \\
\hline Infill Density & $\mathbf{4 0 \%}$ & - \\
\hline Floor Thickness & $\mathbf{1 . 5}$ & $\mathbf{m m}$ \\
\hline Support Angle & $\mathbf{6 8 0}$ & - \\
\hline Support Density & $\mathbf{2 0 \%}$ & - \\
\hline Cooling Fan Speed & $\mathbf{5 0 \%}$ & - \\
\hline Infill Pattern & Linear & - \\
\hline
\end{tabular}

The tensile tests were performed using an Instron electromechanical testing machine D3367 Dual Column System (Instron, United Kingdom) equipped with Bluehill software (Instron, United Kingdom). T he load was measured with a $30 \mathrm{kN}$ load cell having an accuracy of $\pm 0.5 \%$. Each specimen was clamped to the electromechanical testing machine at a specified grip separation of $150 \mathrm{~mm}$ and loaded along the longitudinal axis with a cross-head speed of $2 \mathrm{~mm} / \mathrm{min}$ to failure. The temperature was controlled by an Instron environmental chamber 3119-610 (Instron, United Kingdom). In addition, the temperature of each specimen was also monitored by means of an infrared (IR) camera FLIR TG165 (FLIR Systems, United States) which identifies the temperature distribution in the gage length of each tested specimen. In Figure 4, the gage length of one of the samples tested at $60^{\circ} \mathrm{C}$ is shown. In this Figure, it is possible to see the uniform temperature distribution over the central part of the specimen and a temperature value that differs from the nominal value by $\pm 1^{\circ}$. Although, the temperature displayed on the IR camera is accurate enough on the surface of the specimens, there are some limitations regarding the identification of the temperature profile throughout the thickness of the samples. 


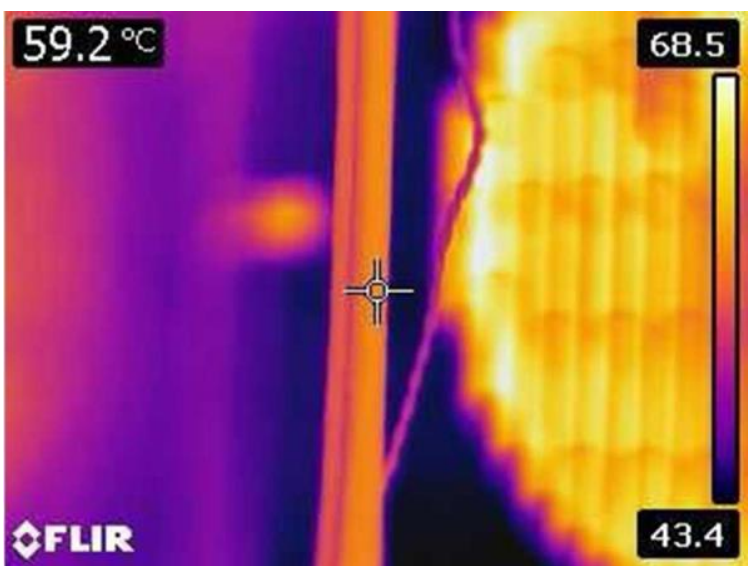

Figure 4 - Temperature contour plot acquired with the IR camera

A preconditioning procedure to record the time needed to reach a specified temperature was carried out. Samples were heated from room temperature to the desired temperature value and the time for each temperature increment was recorded. These times were found to be 3, 7,10 and 15 minutes at the given temperatures of $30^{\circ} \mathrm{C}, 40^{\circ} \mathrm{C}, 50^{\circ} \mathrm{C}$ and $60^{\circ} \mathrm{C}$ respectively.

A Load-displacement curve was acquired during each test which was analysed and later converted into the stress-strain curve. The applied stress was computed as the ratio between the applied load and the initial minimum cross-section area, while the strain was derived by dividing the cross-head displacement over the initial gripping length of the specimen $(150 \mathrm{~mm})$. It is worth mentioning that the calculated minimum cross-section area was based on the externally measured dimensions. However, the potential voids within the built structure are not accounted for in this calculation. The average values of the mechanical properties with the corresponding standard deviation have been computed for each group of 5 specimens considered at a given orientation and temperature. The ultimate tensile strength (UTS), Young's modulus (E), the stress at failure and the strain at failure have been derived in accordance with ASTM: D638 Standard.

Due to the lack in the current literature regarding the effects of the temperature on PLA 3D printed component, it was decided to narrow-down the investigation and focus the attention on the 
combined effect of the infill orientation and the temperature values, while fixing the other parameters.

\subsection{Fractography Analysis of 3D printed specimen}

Fracture surfaces of the tensile test specimens were examined using a LEICA DFC295 digital microscope colour camera (Leica Microsystems, United Kingdom) with a resolution of 2048x1536 pixels (3 Megapixels). The fracture surfaces contain necking as well as voids due to the printing imperfection and the fracture process itself. The fracture surfaces were scanned for these features and as a way of confirming the nature of the failure mechanism. The microscopic observations of the samples tested at different temperature values highlighted porosity due to the printing process as well as the variation in necking. The specimens examined at $50^{\circ} \mathrm{C}$ and $60^{\circ} \mathrm{C}$ did not reach complete separation and were therefore not analysed.

\section{Results and discussion}

\subsection{Filaments}

Tensile tests were carried out on the PLA filament before and after printing. The average curves obtained from the tests performed at the four different temperature values $\left(30^{\circ} \mathrm{C}, 40^{\circ} \mathrm{C}, 50^{\circ} \mathrm{C}\right.$, $\left.60^{\circ} \mathrm{C}\right)$ are reported in Figure 5. Apart from the highest temperature test $\left(60^{\circ} \mathrm{C}\right)$ which did not fail, the remaining specimens failed after their nominal extension had been recorded. 



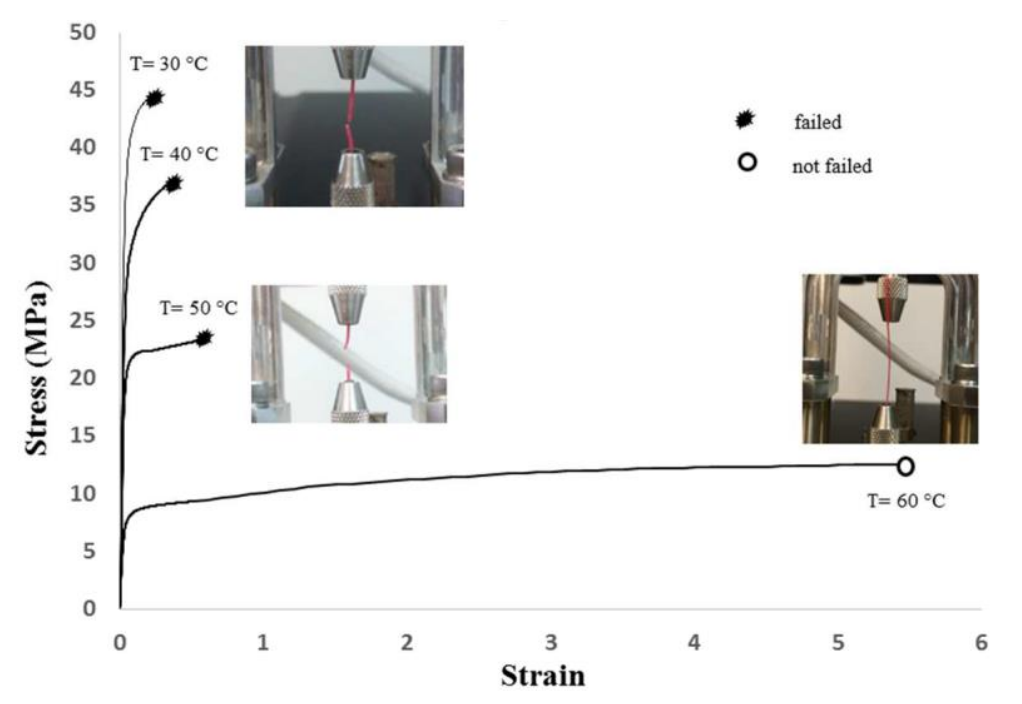

Figure 5 Stress vs strain for printed PLA curve: tensile strength decreased while strain increased as temperature increases

As expected, increasing the temperature results in a significant higher strain at failure. As the temperature increases, the molecular segments motion is triggered enabling free chain movement up to the glass transition phase $(\sim 60 \mathrm{oC})$ which explains the continuous extension of the filament without a defined failure point (Saeidlou, Huneault et al. 2012). Ductile polymers tend to have a well-defined yield point with high strain typically of the order 5-10\% due to their semicrystalline state, whereas most amorphous glassy polymers are typically brittle and rupture at definite low strains.. These effects are generally controlled by the addition of plasticisers or temperature variation (Kendall and Siviour 2014).

Table 3 Experimental result for neat and printed filaments from the tensile test.

\begin{tabular}{c|cc} 
& \multicolumn{2}{|c}{ YOUNG MODULUS [MPA] } \\
\hline TEMPERATURE & NEAT PLA & 3D-PRINTED PLA \\
\hline $\mathbf{3 0}^{\circ} \mathbf{C}$ & $1175 \pm 89$ & $\mathbf{1 4 0 0 \pm 7 8}$ \\
$\mathbf{4 0}^{\circ} \mathbf{C}$ & $1011 \pm 75$ & $\mathbf{1 1 4 3} \pm \mathbf{8 2}$ \\
$\mathbf{5 0}^{\circ} \mathbf{C}$ & $701 \pm 54$ & $\mathbf{9 6 1} \pm \mathbf{5 4}$ \\
$\mathbf{6 0}^{\circ} \mathbf{C}$ & $\mathbf{2 4 7} \pm \mathbf{1 6}$ & $\mathbf{3 5 0} \pm \mathbf{1 2}$
\end{tabular}


Results reported in Table 3 reveal that the thermal history undergone by the polymer during the deposition phase will affect inherently the mechanical property of the final fused (printed) polymer.

For this reason, a modelling approach should take into account the variations on the mechanical properties, from the original feeding material, induced by the printing operations.

According to the tensile stiffness recorded for both plain and 3D printed PLA, it can be observed that there is an inverse relationship between temperature increase and modulus reduction. However, it is worthwhile to mention the comparable reduction in stiffness from $30 \%$ in plain PLA to $16 \%$ for the freely extruded filaments as the temperature increases from $40^{\circ} \mathrm{C}$ to $50^{\circ} \mathrm{C}$. This can be explained by the cold crystallisation effects of such polymers and molecular chain re-alignment along the testing direction (Martin and Avérous 2001). The thermal dependence of PLA properties is explained by the direct influence of its thermal history on the crystallinity of PLA (Farah, Anderson et al. 2016). Therefore, the melting process, within the nozzle, during 3D printing results in the $\alpha$ growth of PLA crystals (Farah, Anderson et al. 2016). The dramatic drop in stiffness as the temperature approaches the glass transition $60^{\circ} \mathrm{C}$, is associated with the glass transition of the polymer into a semi-liquid or rubbery state. This phase is characterised by the expanded free volume which promotes free particles movement and consequently reduces the interlocking mechanism; hence, lowering the measured stiffness (Nikzad, Masood et al. 2011).

\subsection{D printed specimens}

Tensile tests carried out on the 3D printed specimens by means of the electromechanical INSTRON machine, for each group the average over the five values acquired together with the standard deviation is reported. In correspondence to temperature values $50^{\circ} \mathrm{C}$ and $60^{\circ} \mathrm{C}$; the transition in the mechanical behaviour due to the $\mathrm{Tg}$ changes the constraint of the polymeric chains allowing them to move and slide under the action of the applied load. This condition results in a continuous elongation under a constant applied load. Since the failure condition is not reached, the tensile test 
was stopped once a strain of $0.2 \%$ was reached. It has been assumed that this elongation corresponds to the condition for which the functionality of the component is lost, even if separation is not accomplished.

The results presented in Figure 6 indicates the strong influence of temperature increase on both stiffness and ultimate strength UTS of the FDM specimen. It can be clearly observed that as the temperature increases the stiffness and UTS decrease. At temperatures below $40^{\circ} \mathrm{C}$, the stiffness values fluctuate within a narrow range before plummeting at temperatures of $50^{\circ} \mathrm{C}$ and $60^{\circ} \mathrm{C}$ due to the glass transition. However, UTS values experienced a gradual decrease with respect to temperature increase up to $50^{\circ} \mathrm{C}$ at which point a dramatic fall in strength is recorded and continues to drop at $60^{\circ} \mathrm{C}$.

The results acquired at room temperature through tensile tests have confirmed the well-known dependency of the stiffness and strength on the infill orientation (Lanzotti, Grasso et al. 2015). According to the current literature, the maximum value of Young's modulus (E) at room temperature was found in correspondence of $\pm 45^{\circ}$ orientation. This is different to the observed trend found in polypropylene specimens in (Carneiro, Silva et al. 2015) in which the maximum Young's modulus (E) occurred at $0^{\circ} / 90^{\circ}$ orientation. The same response is observed when considering the strength measured as UTS. These observations agree well with the results reported by Dawoud et al., (Dawoud, Taha et al. 2016) in which the tensile strength of ABS, at $\pm 45^{\circ}$ orientation was consistently higher than other orientations used in the study for both positive and negative air gap. However, another study on the influence of build parameters of PEEK samples by (Wu, Geng et al. 2015) on the mechanical properties revealed contradicting results to those reported by Dawoud as the maximum strength was recorded for the $0^{\circ} / 90^{\circ}$ orientation. 
1

2

3

4

5

6

7

8

9

10

11

12

13

14

15

16

17

18

19

23

24

25

26

27

28

29

30

31

32

33

34

35

36

37

38

39

40

41

42

43

44

45

46

47

48

49

50

51

52

53

54

55

56

57

58

59

60
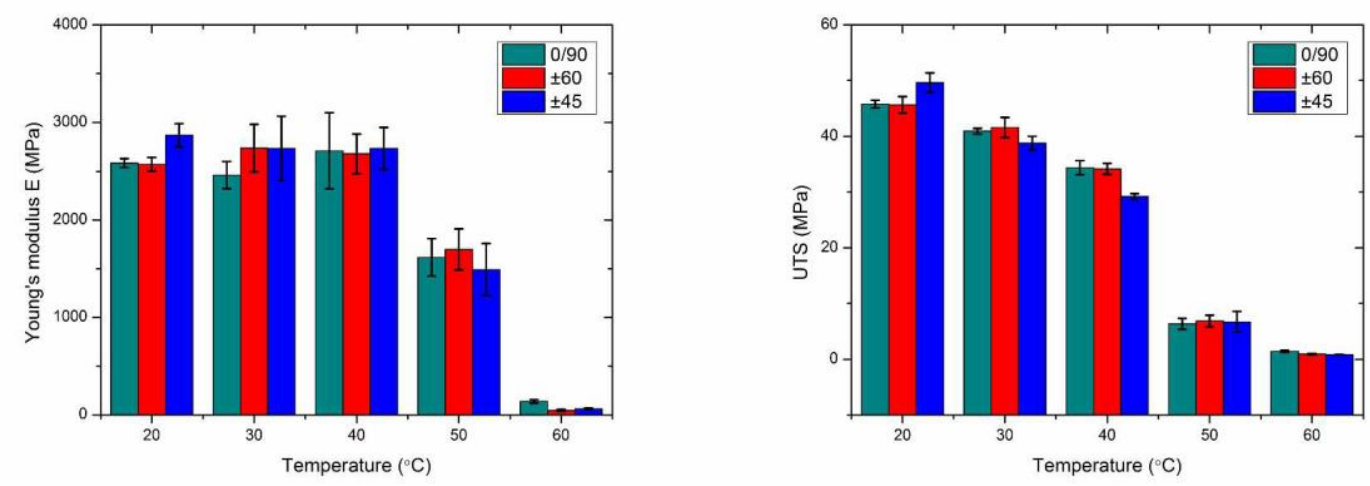

Figure 6 - Young's Modulus (left) and UTS (right) comparison for different infill orientation as temperature increases. 
The difference in the average values of Young's modulus (E) between $0^{\circ} / 90^{\circ}$ and $30^{\circ} / 60^{\circ}$ is 12 $\mathrm{MPa}$. For $0^{\circ} / 90^{\circ}$ the axial load is taken partially by half of the layers oriented along the loading direction and partially by the rest of the layers oriented along the orthogonal direction. The contribution of the latter in sustaining the load is limited to the bonding surfaces that are weaker than the beads. However, the deformability of the cross section is limited hence the brittle failure is dominant as shown in Figure 7a. The irregular shape of the beads, as well as the bonding region between beads, as well as the bonding region between beads locally increases the stress promoting failure. As already described by (Durgun and Ertan 2014), the failure mode is a result of the material separation in a plane approximately normal to the tensile stress. The failure is caused by pulling with high tensile strength and eventual rupture, as the tensile loads were taken by beads themselves.
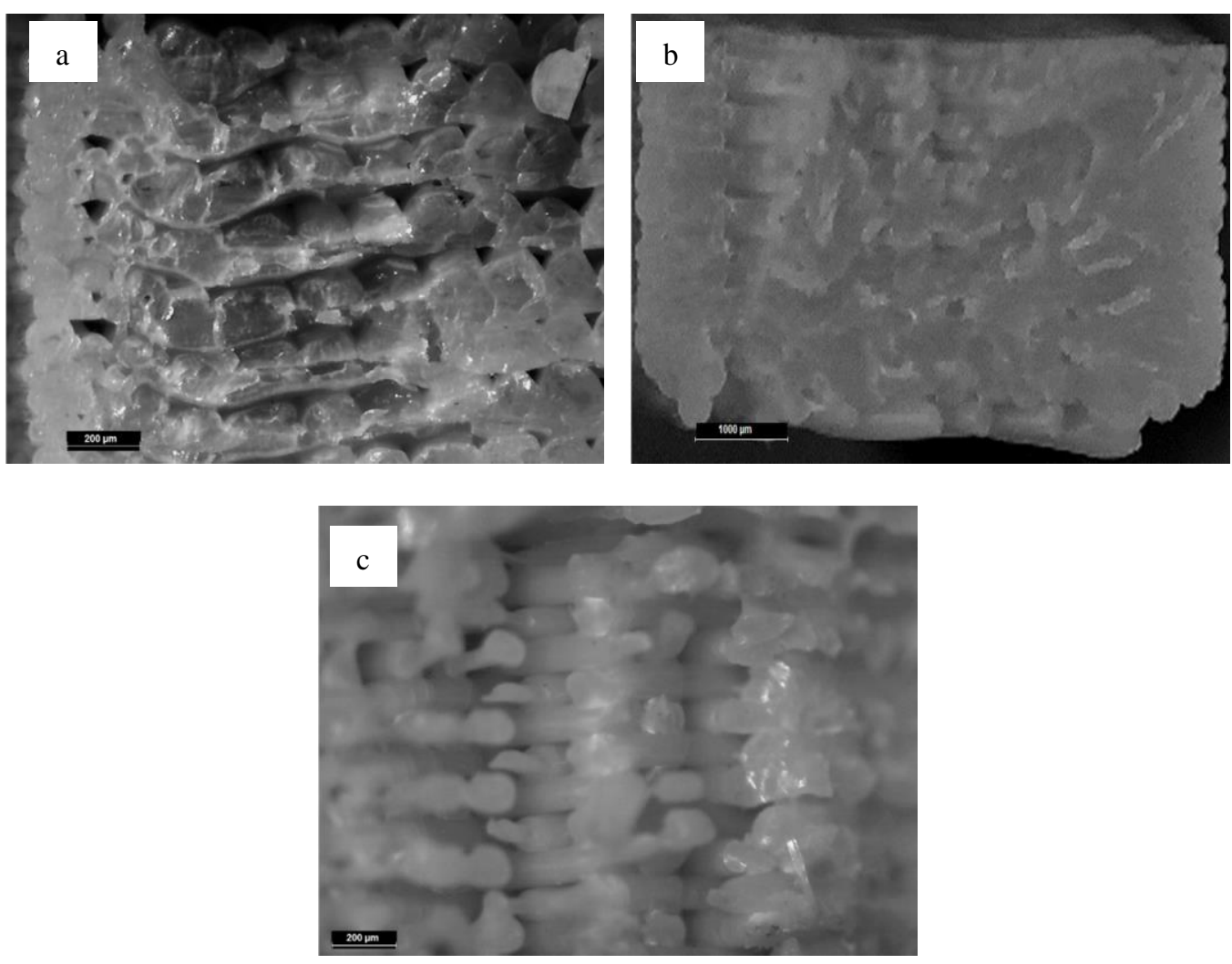

Figure 7 - Microscopic images of specimens printed at: a) $0^{\circ} / 90^{\circ}$, b) $-30^{\circ} / 60^{\circ}$ and c) $\pm 45^{\circ}$ after testing at 
When the beads are oriented along $-30^{\circ} / 60^{\circ}$ in each layer the axial load has a component along the direction of the beads. As seen in Figure $7 \mathrm{~b}$ the presence of more beads that withstand the load compensate the less part of the load taken by the more extended bonding surfaces resulting in a limited reduction of the stiffness value. When the beads are oriented along \pm 45 , the diamond arrangement of the oriented beads (Figure 7c) enables the increased load taken with minimal extension

compared to the two previous orientations, having at the same time a lower extension of the bonding surfaces. Regarding the strength, the minimum UTS value is reached at $-30 \circ / 60$ 。due to the fact that the bonding surfaces play a crucial role in reducing the load sustained by the specimen. At $40{ }^{\circ} \mathrm{C}$ the minimum value of the stiffness is reached at $-30^{\circ} / 60^{\circ}$ and the maximum at $\pm 45^{\circ}$ with a difference between $\pm 45^{\circ}$ and $0^{\circ} / 90^{\circ}$ of $26 \mathrm{MPa}$. The minimum value of the UTS is reached at $\pm 45^{\circ}$ and the maximum at $0^{\circ} / 90^{\circ}$. The higher stiffness value at $\pm 45^{\circ}$ corresponds to a lesser value of the strength with the minimum value of the UTS. For all other orientations, necking plays a major role in the failure mechanism resulting in an immediate failure after the UTS is reached. The tests carried out at $40^{\circ} \mathrm{C}$ have shown a failure mode mainly ductile with necking in the beads and visible necking in the cross-section of the specimens. 

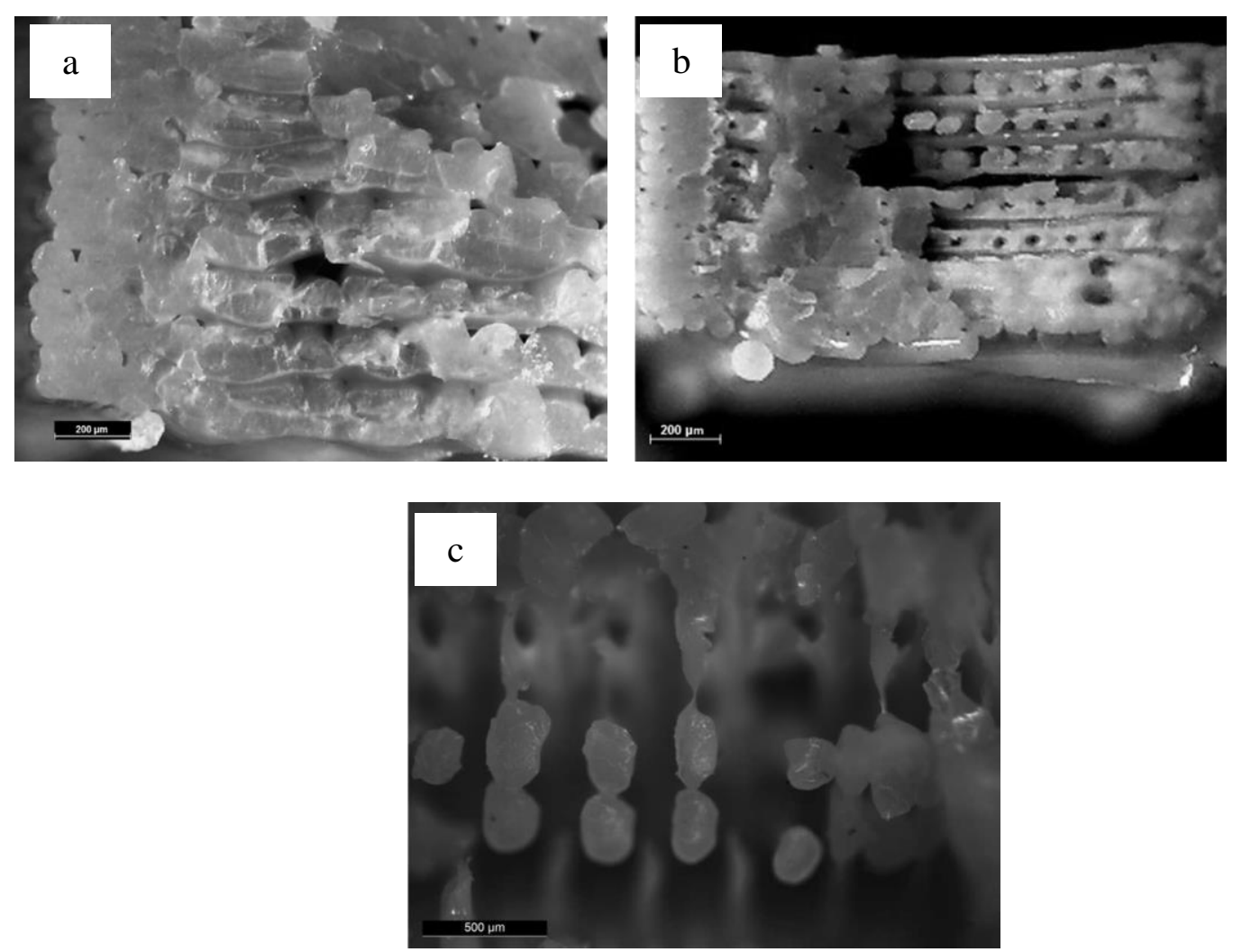

Figure 8 - Microscopic images of specimens printed at: a) $\left.0^{\circ} / 90^{\circ}, b\right)-30^{\circ} / 60^{\circ}$ and c) $\pm 45^{\circ}$ after testing at

$40^{\circ} \mathrm{C}$.

The combination of infill orientation and temperature results in the failure of the beads. The combination of infill orientation and temperature trigger the shear failure of the samples. Shear is induced by the force acting in parallel planes, consequently sliding movement of beads layers along the build direction as depicted in Figure 9. Bonding surfaces are the contact regions created among adjacent beads. In fact, when all beads are broken, the load is carried mainly by the bonding surfaces which will tend to creep along their directional planes due to shear forces. Moreover, the inspection of the fracture surfaces (Figures $8 \mathrm{a}, \mathrm{b}$ and c) of the specimens together with the analysis of the stress-strain curves reveal that the response is characterized by evident softening due to the shear response resulting in a higher strain at failure. The effects of the temperature promote creep which contributes to the greater elongation (Yang, Zhang et al. 2006). 

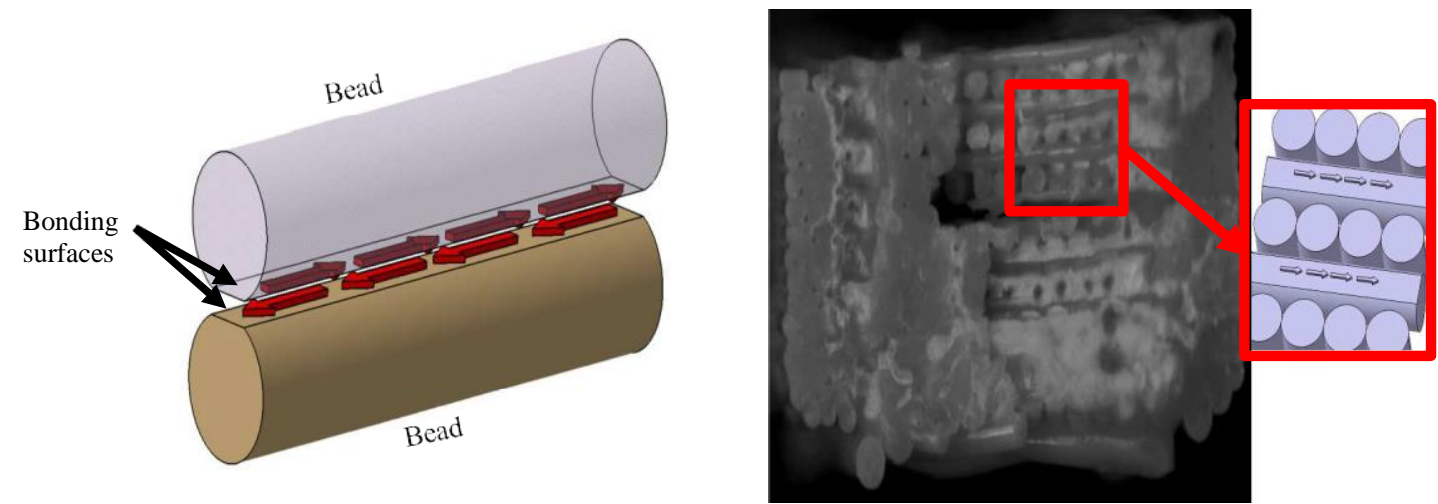

Figure 9 Sliding movement of bonding surfaces under shear stress (left), visual and representative

At $50^{\circ} \mathrm{C}$ there is a drop in the stiffness values for $0^{\circ} / 90^{\circ}$ and $\pm 45^{\circ}$ which are $1100 \mathrm{MPa}$ and 1250 MPa respectively. At $50{ }^{\circ} \mathrm{C}$, the effect of temperature on the $-30^{\circ} / 60^{\circ}$ is different due to the different contribution from the beads and the bonding surfaces. In this particular case, most of the beads are oriented closer to the loading line resulting in most of the load being taken by the beads rather than the bonding surfaces.

At $60^{\circ} \mathrm{C}$, a value that is greater than the $\mathrm{Tg}$, the values for Young's modulus (E) are considerably low for all considered orientations. Below this temperature, any dimensional changes are dominated by the temporary distortions of the primary valence bonds. As polymers are either semi-crystalline or amorphous, the resulted structure after extrusion is considered to be in the semicrystalline phase, with repeating crystals, which is responsible for the higher tensile strength and stiffness. These properties decrease drastically above the $\mathrm{T}_{\mathrm{g}}$ (Van de Velde and Kiekens 2002). However, it is still possible to identify the difference between the three orientations, and in particular, the maximum value is reached at $0^{\circ} / 90^{\circ}$ whilest the minimum at $-30^{\circ} / 60^{\circ}$.

Regarding stress at failure, the maximum value at room temperature was recorded at $0^{\circ} / 90^{\circ}$ while the minimum occurred at $\pm 45^{\circ}$. A general trend is observed in Figure 10, increasing the temperature value causes reduction of the stress at failure for the $0^{\circ} / 90^{\circ}$ and $-30^{\circ} / 60^{\circ}$. As observed with the optical 
microscope in Figure 8c, the extended necking, plastic deformation and the brittleness of the bonding surfaces, which are playing a predominant role for this orientation, are affecting the beads as well as the entire cross-section. On the other hand, for the other two orientations the contribution from the beads is predominant compared to the bonding surfaces, and consequently, the stress at failure is much higher. For the tests carried out at $50^{\circ} \mathrm{C}$ and $60^{\circ} \mathrm{C}$, the stress of failure is identified as the constant stress under which the material flow at high strain values.
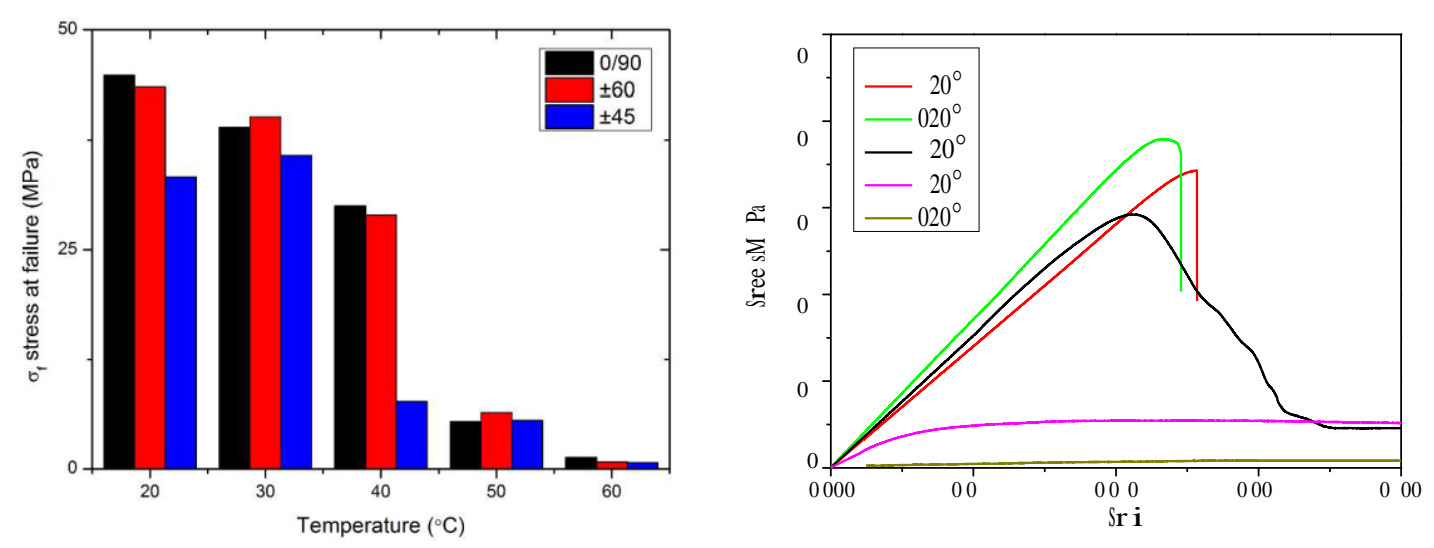

Figure 10 Stress at failure as a function of temperature (left): stress at failure decreases as temperature increases for all infill orientations, Stress vs strain curves of $\pm 45^{\circ}$ samples (right)

The strain at failure at $50^{\circ} \mathrm{C}$ and $60^{\circ} \mathrm{C}$ were evaluated at $0.2 \%$ since failure with the complete separation of the two ends of the sample was never reached. For that reason, it is not possible to make any comparisons. At room temperature, the maximum strain at failure is reached at $30 \% 60^{\circ}$ as the overall longitudinal strain is mainly composed by the elongation of the beads along the loading line. The lowest strain at failure at $20{ }^{\circ} \mathrm{C}$ occurs at $\pm 45^{\circ}$, since the strain is mostly contributed by the bonding surfaces without any significant axial elongation of the beads.

As the temperature increases to $40^{\circ} \mathrm{C}$, the highest strain at failure value is reached at $\pm 45^{\circ}$ whereas the lowest value is at $-30^{\circ} / 60^{\circ}$. The combination of bonding surfaces deformation with the 
axial elongation of the beads can also explain the different behaviour at that temperature value. The increase in temperature is causing a drop in the shear strength of the bonding surface among beads. As a result, the load is mainly carried by the beads since the resistance opposed by the bonding surfaces is minimal. The more load carried by the beads results in more elongation and a dominant necking all over the cross-section.

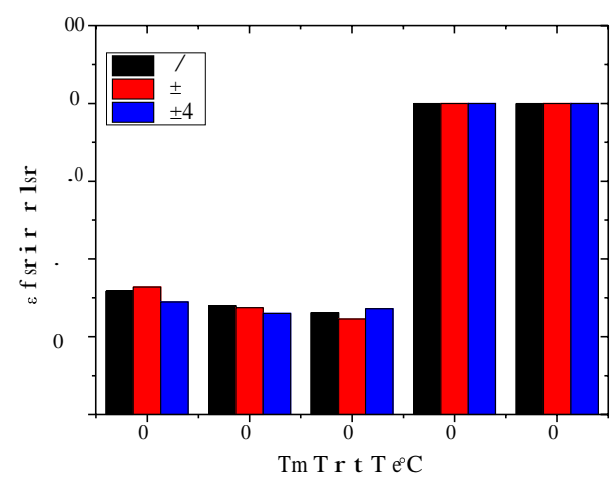

Figure 11 Strain at failure for different infill orientations as a function oftemperature

\section{Conclusion}

In this paper, the effects of the temperature on the mechanical response of PLA 3D printed specimens was studied. The standard process parameters were changed to identify the correlations between the printing parameters and the temperature values. The response surfaces were used to derive the required relationship among process parameters, temperature, the tensile stiffness, the UTS, the strain and stress at failure. The analysis of the experimental results made it possible to understand the impact of control factors on the mechanical properties of specimens produced using the Fusion Additive Manufacturing. The tensile tests carried out on single filaments before and after the extrusion process with the 3D printer have shown a reduction in stiffness from $30 \%$ in plain PLA filaments to $16 \%$ in $3 \mathrm{D}$ printed filament as temperature increases from $40{ }^{\circ} \mathrm{C}$ to $50{ }^{\circ} \mathrm{C}$. This was explained by the cold crystallisation effects of such polymers and molecular chain realignment along the testing direction. Furthermore, the results of the tensile tests carried out on the 
$3 \mathrm{D}$ printed samples at different temperature values were reported and discussed. With regard to Young's modulus (E) values, it is possible to observe a common trend for $0^{\circ} / 90^{\circ}$ and $\pm 45^{\circ}$ as the temperature increases, while for $-30^{\circ} / 60^{\circ}$ at $50^{\circ} \mathrm{C}$ it is following a different path presenting a higher magnitude when compared to the other two orientations. Regarding the UTS, it is possible to identify a continuous reduction in values as the temperature increases and the three configurations follow the same path. The stress at failure for the $\pm 45^{\circ}$ orientation is showing a peculiar phenomenon with the maximum value at $30^{\circ} \mathrm{C}$ and a sudden drop at $40^{\circ} \mathrm{C}$; this latter is higher than what was observed for the other two orientations. At $+/-45^{\circ}$, the temperature variation from $20^{\circ} \mathrm{C}$ to $30^{\circ} \mathrm{C}$ lowers the strength of the bonding surface which is counterbalanced by the beads robustness into the overall force loading balance of the sample. At a higher temperature, however, closer to the glass transition temperature of the PLA, (i.e. $40^{\circ} \mathrm{C}$ ), the weakness of the beads induces a redistribution of the stresses to determine a magnitude level at bonding surface which exceeds the shear strength of the interface determine the failure. For $0^{\circ} / 90^{\circ}$ and $-30 \% / 60$ othe stress at failure decrease as the temperature increases with a consistent reduction at $50^{\circ} \mathrm{C}$ which is close to the $\mathrm{Tg}$ for PLA. Regarding the strain at failure, it was observed that the combination of bonding surfaces deformation with the axial elongation of the beads can explain the different behaviours of the three orientations here investigated as the temperature values increase. There is potential for further analyses of the thermomechanical properties of FDM parts using finite element analysis methods to model the material properties based on the experimental stress-strain results.

\section{References}

Ahn, S.-H., M. Montero, D. Odell, S. Roundy and P. K. Wright (2002). "Anisotropic material properties of fused deposition modeling ABS." Rapid prototyping journal 8(4): 
248-257.

Bellini, A. and S. Güçeri (2003). "Mechanical characterization of parts fabricated using fused deposition modeling." Rapid Prototyping Journal 9(4): 252-264.

Bikas, H., P. Stavropoulos and G. Chryssolouris (2016). "Additive manufacturing methods and modelling approaches: a critical review." The International Journal of Advanced Manufacturing Technology 83(1-4): 389-405.

Bito, J., R. Bahr, J. G. Hester, S. A. Nauroze, A. Georgiadis and M. M. Tentzeris (2017). "A Novel Solar and Electromagnetic Energy Harvesting System With a 3-D Printed Package for Energy Efficient Internet-of-Things Wireless Sensors." IEEE Transactions on Microwave Theory and Techniques.

Carneiro, O. S., A. F. Silva and R. Gomes (2015). "Fused deposition modeling with polypropylene." Materials \& Design 83: 768-776.

Dawoud, M., I. Taha and S. J. Ebeid (2016). "Mechanical behaviour of ABS: An experimental study using FDM and injection moulding techniques." Journal of Manufacturing Processes 21: 39-45.

de Obaldia, E. E., C. Jeong, L. K. Grunenfelder, D. Kisailus and P. Zavattieri (2015). "Analysis of the mechanical response of biomimetic materials with highly oriented microstructures through 3D printing, mechanical testing and modeling." Journal of the mechanical behavior of biomedical materials 48: 70-85.

Durgun, I. and R. Ertan (2014). "Experimental investigation of FDM process for improvement of mechanical properties and production cost." Rapid Prototyping Journal 20(3): 228-235.

Farah, S., D. G. Anderson and R. Langer (2016). "Physical and mechanical properties of PLA, and their functions in widespread applications - A comprehensive review." Advanced Drug Delivery Reviews 107: 367-392.

Feng, S., D. Wu, H. Liu, C. Chen, J. Liu, Z. Yao, J. Xu and M. Zhang (2014). "Crystallization and creep of the graphite nanosheets based poly(butylene adipate-coterephthalate) biocomposites." Thermochimica Acta 587(Supplement C): 72-80.

Gibson, I., D. W. Rosen and B. Stucker (2010). Additive manufacturing technologies, Springer. 
Hong, A. L., B. T. Newman, A. Khalid, O. M. Teter, E. A. Kobe, M. Shukurova, R. Shinde, D. Sipzner, R. J. Pignolo and J. K. Udupa (2017). Feasibility of fabricating personalized 3D-printed bone grafts guided by high-resolution imaging. SPIE Medical Imaging, International Society for Optics and Photonics.

Impens, D. and R. Urbanic (2016). "A comprehensive assessment on the impact of postprocessing variables on tensile, compressive and bending characteristics for 3D printed components." Rapid Prototyping Journal 22(3): 591-608.

Keles, O., C. W. Blevins and K. J. Bowman (2017). "Effect of build orientation on the mechanical reliability of 3D printed ABS." Rapid Prototyping Journal 23(2).

Kendall, M. J. and C. R. Siviour (2014). Rate dependence of poly (vinyl chloride), the effects of plasticizer and time-temperature superposition. Proc. R. Soc. A, The Royal Society.

Lanzotti, A., M. Grasso, G. Staiano and M. Martorelli (2015). "The impact of process parameters on mechanical properties of parts fabricated in PLA with an open-source 3-D printer." Rapid Prototyping Journal 21(5): 604-617.

Li, J., B. Wu and C. Myant (2016). "The Current Landscape for Additive Manufacturing Research."

Martin, O. and L. Avérous (2001). "Poly(lactic acid): plasticization and properties of biodegradable multiphase systems." Polymer 42(14): 6209-6219.

Moroni, L., J. De Wijn and C. Van Blitterswijk (2006). "3D fiber-deposited scaffolds for tissue engineering: influence of pores geometry and architecture on dynamic mechanical properties." Biomaterials 27(7): 974-985.

Nikzad, M., S. Masood and I. Sbarski (2011). "Thermo-mechanical properties of a highly filled polymeric composites for fused deposition modeling." Materials \& Design 32(6): 3448-3456.

Qin, Z., B. G. Compton, J. A. Lewis and M. J. Buehler (2015). "Structural optimization of 3D-printed synthetic spider webs for high strength." Nature communications 6.

Rodríguez, J. F., J. P. Thomas and J. E. Renaud (2001). "Mechanical behavior of acrylonitrile butadiene styrene (ABS) fused deposition materials. Experimental investigation." Rapid Prototyping Journal 7(3): 148-158. 
Saeidlou, S., M. A. Huneault, H. Li and C. B. Park (2012). "Poly(lactic acid) crystallization." Progress in Polymer Science 37(12): 1657-1677.

Senatov, F., K. Niaza, M. Y. Zadorozhnyy, A. Maksimkin, S. Kaloshkin and Y. Estrin (2016). "Mechanical properties and shape memory effect of 3D-printed PLA-based porous scaffolds." Journal of the mechanical behavior of biomedical materials 57: 139-148.

Shakor, P., J. Sanjayan, A. Nazari and S. Nejadi (2017). "Modified 3D printed powder to cement-based material and mechanical properties of cement scaffold used in 3D printing." Construction and Building Materials 138: 398-409.

Thomas, J. P. and J. E. Renaud (2003). "Design of fused-deposition ABS components for stiffness and strength." Journal of Mechanical Design: 545-551.

Torres, J., M. Cole, A. Owji, Z. DeMastry and A. P. Gordon (2016). "An approach for mechanical property optimization of fused deposition modeling with polylactic acid via design of experiments." Rapid Prototyping Journal 22(2): 387-404.

Torres, J., J. Cotelo, J. Karl and A. P. Gordon (2015). "Mechanical Property Optimization of FDM PLA in Shear with Multiple Objectives." Jom 67(5): 1183-1193.

Van de Velde, K. and P. Kiekens (2002). "Biopolymers: overview of several properties and consequences on their applications." Polymer Testing 21(4): 433-442.

Visscher, D. O., E. J. Bos, M. Peeters, N. V. Kuzmin, M. L. Groot, M. N. Helder and P. P. van Zuijlen (2016). "Cartilage tissue engineering: Preventing tissue scaffold contraction using a 3D-printed polymeric cage." Tissue Engineering Part C: Methods 22(6): 573-584.

Wu, W., P. Geng, G. Li, D. Zhao, H. Zhang and J. Zhao (2015). "Influence of layer thickness and raster angle on the mechanical properties of 3D-printed PEEK and a comparative mechanical study between PEEK and ABS." Materials 8(9): 5834-5846.

Yang, J.-L., Z. Zhang, A. K. Schlarb and K. Friedrich (2006). "On the characterization of tensile creep resistance of polyamide 66 nanocomposites. Part I. Experimental results and general discussions." Polymer 47(8): 2791-2801. 